\title{
A TRANSGRESSÃO DA CULTURA POPULAR SUL -COREANA: A HALLYU E SUA INFLUÊNCIA NO CONSUMO DE PRODUTOS SUL- COREANOS
}

\author{
TRANSGRESSION OF SOUTH KOREAN POP CULTURE: Hallyu Influences \\ The Consumption Of South Korean Products
}

\author{
Marina Presser Alves da Silva ${ }^{1}$ \\ Amália Costa Farias ${ }^{2}$
}

RESUMO: Este artigo tem o intuito de examinar a preferência por produtos coreanos ou adjuntos à cultura coreana, a partir da crescente popularidade do fenômeno da $h a l l y u^{3}$. A música popular coreana conhecida por $k-p o p^{4}$ e as telenovelas, denominadas $k$-dramas 5 , não chegaram ao Brasil influenciados por mediação de grandes empresas de disseminação de mídia como geralmente acontece, mas sim por meio da internet (URBANO, 2017). Ao longo do tempo o entretenimento sul coreano vem sendo disseminado por comunidades virtuais que realizam traduções e distribuição, além de discutir o assunto entre si. Por meio de um levantamento bibliográfico e jornalístico, discutiremos o potencial de influência do consumo de produtos coreanos. Também serão analisados os resultados de uma pesquisa qualitativa utilizando a metodologia de análise de conteúdo proposta por Bardin, considerando palavras dos relatos para compreender a relação do público com o objeto de estudo, em seguida abordaremos a perspectiva de Canclini sobre consumo como forma de expressão transcultural, para então discutir a possível tangibilização da experiência proposta no entretenimento coreano por meio do consumo de produtos. Nesse sentido, consideramos a hallyu como um fenômeno cultural onde o entretenimento apresenta de forma contextualizada os produtos industrializados ${ }^{6}$ aos espectadores não-coreanos.

\footnotetext{
I Graduanda em Comunicação Social em Publicidade e Propaganda pela Universidade Anhembi Morumbi (UAM/SP) http://lattes.cnpq.br/7792518328111009

${ }^{2}$ Mestrado em Administração pela Pontifícia Universidade Católica de São Paulo (PUC/SP) Docente pela Universidade Anhembi Morumbi (UAM/SP) http://lattes.cnpq.br/3805636940894782

${ }^{3}$ Hallyu, traduzido como "Onda Coreana", é o termo que contempla o conjunto elementos da cultura coreana que se popularizaram pelo mundo. Conforme definição disponibilizado pelo Ministry of Culture, Sports and Tourism and Korean Culture and Information Service, o termo surgiu nos anos 90 quando a cultura coreana se tornou popular na China devido à exportação de telenovelas coreanas.

${ }^{4} \mathrm{~K}$-pop é uma abreviação de korean popular music, que é a música popular coreana contemporânea, o termo não possui viés de cultura popular tradicional.

${ }^{5} \mathrm{~K}$-dramas é uma abreviação para korean dramas, são as telenovelas coreanas.

${ }^{6}$ Pela perspectiva do sistema tributário brasileiro, o regulamento de imposto sobre produto industrializado, se define como produto industrializado aquele que é obtido por meio de um processo
} 
Palavras-chave: Hallyu; Coréia do Sul; Consumo; Transcultura

ABSTRACT: This article aims to understand preference for korean products related to korean culture, based on the growing popularity of the hallyu phenomenon. Korean popular music also known as $k$-pop, and korean soap operas known as k-dramas did not arrive in Brazil by major media companies as usually happens, but through the internet (URBANO, 2017). South Korean entertainment has been popular by virtual communities that translate and distribute all this material, in addition to discussing with members of this community. Through a bibliographic and journalistic research, we will discuss the potential influence of consumption of korean products in Brazil. Will be also considered the results of a qualitative research for this analysis using content analysis methodology proposed by Bardin, considering customer sentiment through key words, then we will approach Canclini's perspective on consumption as a form of cross-cultural expression, to discuss the possible tangibilization of experiences that is presented by south korean entertainment, through consumption of products. In this sense, we considered hallyu as a cultural phenomenon where entertainment presents industrialized products with contextualization to non-korean audiences.

Keywords: Hallyu; South Korean; Consumption; Transcultural.

\section{INTRODUÇÃO}

O conceito de transgressão ao que se refere ao fenômeno do mar chegar ao solo, inundando o continente terrestre, se torna uma referência à tradução literal da palavra hallyu: a onda coreana.

No Brasil o evento que marcou o potencial da cultura coreana foi a vinda do grupo MBLAQ na cidade de São Paulo em 2orı, como jurados em uma competição de cover de $k$-pop, foi um evento onde grupos se apresentaram reproduzindo a coreografia de uma performance de $k$-pop de sua preferência, e os vencedores teriam a oportunidade de participar de uma competição mundial, concorrendo a chance de ir para a Seul na Coreia do Sul. A própria ação de trazer um grupo foi uma estratégia que buscava a participação do público ao evento, mas a dimensão dada pela produção do evento diante a realidade foi inesperadamente desproporcional, milhares de pessoas se

ou operação, sendo feito por inteiro ou parcial, a ponto de modificar a natureza, finalidade ou aperfeiçoamento para o consumo. 
aglomeraram na Avenida Paulista na cidade de São Paulo (URBANO, 2017). A partir desse evento se dá início a uma série de eventos que contaram com apoio do próprio governo sul-coreano, onde tivemos um aumento gradativo da participação do público a cada ano, chegando ao público de 85 mil pessoas para dois dias de concerto do grupo de k-pop BTS no ano de 2019, também na cidade de São Paulo.

O cenário da música pop coreana é apenas uma parte que pertence ao todo da onda coreana, que é composta por uma variedade de produtos, desde entretenimento, tecnologia, cosméticos e alimentos que conversam entre si, e possuem traços que expressam sua cultura, como hábitos alimentares, vestimenta, história e arte. Historicamente o que dá origem ao termo hallyu é a popularização dos dramas ${ }^{7}$ coreanos na China, sendo as telenovelas o produto vanguardista de exportação da onda coreana pelo continente asiático, enquanto no Brasil, o conteúdo também tem seu público que consome diversas séries e filmes legendados em portugês por meio de fansub ${ }^{8}$, sendo atualmente reconhecidos como produto rentável a partir da disponibilização em plataformas de streaming 9 como a Netflix.

O serviço faz parte da história recente dos produtos audiovisuais coreanos, a marca flerta com fãs da hallyu nas redes sociais por meio de postagens com interação direta aos conteúdos de dramas coreanos, e até mesmo inserindo músicas coreanas em outras séries ocidentais como o caso do teaser $^{10}$ da série Enola Holmes, onde aparece a canção "Señorita" do grupo G-IDLE, ainda que de forma tímida são inserções de pontos da hallyu que começam a aparecer para o público brasileiro consumidor da plataforma.

\section{A patente cultural sul coreana}

Para compreender como esse fenômeno atravessou o mundo e chegou no Brasil independente de barreiras linguísticas e culturais é necessário olhar para a história que

\footnotetext{
7 Dramas é como são chamadas as novelas coreanas, geralmente televisionadas e podendo ter formatos em capítulos semelhantes às novelas brasileiras.

${ }^{8}$ O termo fansub é uma abreviação para "fan subtitle" que se refere aos materiais audiovisuais legendados e distribuídos por um grupo de fãs, geralmente por meio de fórum virtual.

${ }^{9}$ Streaming (inserir contexto)

ro Teaser
} 
a Coréia do Sul viveu e construiu para si e para o mundo. A Coréia do Sul em relação aos países asiáticos foi no passado considerado uma "cultura satélite" desprendido ao mesmo tempo dependente de países maiores como a China, a península teve ao longo de sua história relações diplomáticas e econômicas com China, chegando a contribuir com o sistema de impostos em troca de apoio e defesa da região. Posteriormente a península é ocupada pelo império japonês que se estabelece durante anos, impondo adoção da cultura japonesa como forma de opressão e dominação territorial e cultural, que só termina após a derrota dos aliados na Segunda Guerra Mundial (SETH, 2009). A partir de então se inicia o momento de recuperação da cidadania e identidade coreana, que após tantos anos suprimida busca como sua primeira referência a própria cultura tradicional. No que se entendem por arte tradicional coreana, a principal referência vem de seus antepassados da Dinastia Joseon ${ }^{\text {II }}$ (I392 a I897), considerada a última dinastia de sua história. O período foi considerado como a mais longa dinastia confucionista $^{12}(\mathrm{SETH}$, 2009), que exerceu influências sociais e culturais importantíssimas como a criação do alfabeto coreano, uma vez que se utilizavam caracteres chineses para composição escrita do idioma coreano (SETH, 2009). Quando o governo retoma o controle de suas narrativas e decide deixar de ser um satélite é que se dá início ao que temos como a Hallyu (KIM, 2015), um movimento mais contemporâneo de expressão cultural.

Os elementos culturais tradicionais são trazidos no entretenimento de forma constante dentro de uma estratégia de diferenciação e destaque perante aos países vizinhos, e é por meio do entretenimento que contam sua história escolhendo quais sentimentos desejam passar para o mundo, uma espécie de controle de sua própria narrativa (MANZUR, 2014) enquanto nação. O que define a cultura sul coreana como relevante no cenário globalizado são justamente os desdobramentos do entretenimento para desde o consumo de produtos industrializados, até mesmo questões políticas,

\footnotetext{
"Joseon (no alfabeto coreano 조선, pronúncia “choson"), considerado a última dinastia da Coreia como um único território, para saber mais detalhes sobre as características do período, visitar http://www.korea.net/AboutKorea/History/Joseon acesso em 9 nov. 2020

${ }^{12}$ Confucionismo é o nome dado para linha filosófica de Confúcio (552 a.c a 489 a.c), considerada uma linha de pensamento orientada para a moral individual e governamental.
} 
econômicas, e sociais advindas desse entretenimento.

O início da expansão da Onda Coreana pela Ásia foi liderado pelos dramas televisivos[...]. Vencendo aos poucos as barreiras linguísticas, políticas e culturais, os k-dramas abriram caminho para a divulgação da música pop sul-coreana através de suas trilhas sonoras e, mais tarde, com seus cantores-atores. Essas duas vertentes do fenômeno Hallyu dialogam entre si e, juntas, difundem uma noção do que é "ser coreano. [...]. MANZUR, 2014

$\mathrm{Na}$ década de 60 o grupo de países conhecidos por tigres asiáticos ${ }^{13}$ detinham a maior participação do mercado de exportação em diversos setores da indústria de manufatura, que os colocou nos principais indicadores econômicos mundiais, a alavancada do país deu margem para que, com poder financeiro, pudesse planejar os próximos passos e adquirir recursos estruturais do país (MINNS, 2020).

As transformações tecnológicas e evoluções ao longo do tempo culminaram no que conhecemos por indústria $4.0^{14}$, seus desdobramentos são os mais diversos no que se refere a consumo, uma vez que se trata de um modelo com maior interatividade entre usuário e produtos/serviços (HERMAN; PENTEK; OTTO, 2015). Porém, uma crise econômica generalizada pela saturação de ofertas da indústria de manufatura asiática em 1997 obriga o país à uma maior abertura de mercado conforme acordo realizado com o $\mathrm{FMI}^{15}$ colocando num cenário de repensar seus próximos passos para retomada econômica, situação na qual se gera uma preocupação sobre a influência estrangeira não só em aspectos econômicos, mas também culturais, preocupação adquirida após anos sob o domínio do império japonês. A forma pela qual o governo buscou reconstruir sua economia e desenvolver políticas públicas centradas no fortalecimento da indústria audiovisual nos últimos anos são exemplos de seu grande

\footnotetext{
${ }^{13}$ Tigres asiáticos são o grupo de países dominantes do modelo de industrialização para exportação, que levou os países à altos patamares econômicos, tal acontecimento também teve influência de investimento econômico dos EUA que por sua vez não hesitou em manter territórios com economia capitalista como forma de recuar os avanços do socialismo durante a guerra fria.

${ }^{14}$ Conceito apresentado na feira de Hannover na Alemanha, com o propósito de aumentar a participação de mercado nas indústrias de exportação. https://www.europarl.europa.eu/RegData/etudes/BRIE/2015/568337/EPRS_BRI(2015)568337_EN.pdf is Fundo Monetário Internacional, organização financeira responsável pelos principais indicadores econômicos e apoio financeiro a diferentes países, em troca desse apoio, a organização passa orientações sobre mudanças que devem ser realizadas para recuperação econômica do país.
} 
domínio sobre o poder brando da influência cultural (NYE, 2004), sendo o sucesso dos artistas, cineastas e celebridades não uma mera coincidência, mas sim resultado de ações planejadas.

A preocupação em fortalecer economicamente e culturalmente é expressa nos anos seguintes, o governo começa a desenvolver e controlar seus poderes por meio de políticas públicas centradas no fortalecimento da indústria do entretenimento como um todo. Em 1998 o governo do Presidente Kim Dae Jung chegou a banir a cultura pop japonesa que estava em alta em todo o mundo (JOO. 20II) como forma de protecionismo, perpetuando uma preocupação dos líderes do Estado em não se tornarem influenciáveis culturalmente, seja pela cultura japonesa, chinesa, e até mesmo a estadunidense, (JOO. 20II) e por meio de investimentos em parceria público e privado começam surgir diversos incentivos à produção de entretenimento sul coreano permitindo o crescimento, escalabilidade e distribuição da indústria audiovisual.

No Brasil durante muitos anos a distribuição de produtos midiáticos asiáticos, com exceção das animações japonesas, foi até pouco tempo negligenciada pelas grandes mídias (URBANO, 2017), sendo os fãs responsáveis pela reprodução dos primeiros conteúdos em português, e assim desenvolvendo uma forte comunidade responsável por traduzir, legendar e distribuir à sua maneira esse tipo de mídia (JENKINS, 2008). Tal comportamento também é observado em relação a alguns produtos de bens de consumo denominados como fanmade ${ }^{16}$, que caracterizam produtos feitos por fãs e não endossados por uma marca ou pelos próprios artistas coreanos.

A ausência de distribuição de entretenimento bem como de produtos licenciados e oficiais desses artistas no Brasil impulsiona a existência de produtos como camisetas e artigos de papelarias produzidos por pequenas lojas e por fãs, esse tipo de produto é muito comum e presente na cena de fãs do k-pop, conforme um dos relatos de um participante da pesquisa: "Comprei produtos licenciados de idols, camisetas

\footnotetext{
${ }^{16}$ Fanmade traduzido do inglês "feito por fã", carrega consigo a ideia de um produto personalizado com características e linguagem própria sem intenção de divulgação comercial mas sim de adoração por parte de quem gosta, de fã para fã.
} 
fanmade e produtos de beleza de marca coreana. É inevitável não fazer a associação com $k$-pop, primeiro pela natureza do primeiro e do segundo produto que comprei, e segundo, pela propaganda boca-a-boca que recebi do terceiro produto". É possível por meio da fala notar a diferença entre produtos licenciados e camisetas fan made, demonstrando conhecimento da diferença dos produtos, segundo Canclini (1997) a lógica que rege a interação no consumo de produtos não se trata de satisfazer uma necessidade apenas, mas sim da escassez e impossibilidade de compra por outros, tornando algo exclusivo e identificável por pessoas que pertencem a esse grupo de fãs, principalmente para produtos licenciados e originais que demonstram uma conexão forte pelo seu valor agregado de marca (KOTLER; KELLER, 2006, p.270) ${ }^{17}$.

Foi possível observar que o produto por si só é capaz de gerar interesse no consumo ao ponto de que, mesmo que com pouca participação no mercado brasileiro, os próprios consumidores personalizam diversos itens que estampam artistas e referências sul coreanas como uma forma de suprir a ausência de produtos que dão continuidade no ciclo de consumo, que se inicia com a mídia e o entretenimento, levando aos produtos industrializados.

\section{Desdobramentos publicitários e campanhas globais}

Quando grandes marcas começaram a incorporar artistas, elementos e características da hallyu a fins de atrair o público, a cultura pop coreana se afirma como um fenômeno reconhecido a um grande público consumidor, no qual compensa as marcas a realizarem investimentos publicitários direcionado à esse público, a publicidade e endosso de marcas se torna o termômetro de tendências ao sucesso. Não tão coincidentemente, o também sucesso que ultrapassou barreiras culturais levando consigo prêmios consagrados, o sucesso do filme Parasita (2019) de Bong Joon Ho rendeu desdobramentos publicitários transnacionais. Em uma cena específica do filme, os personagens da família Park ligam para sua governanta avisando que sua viagem

\footnotetext{
${ }^{17}$ Brand equity, ou equidade de marca, para Kotler e Keller representa a imagem favorável do consumidor à marca, sendo um valor agregado atribuído a produtos e serviços indispensável para fortalecimento da marca.
} 
foi cancelada e que estão no caminho de volta pra casa, ao retornar pedem que a mesma prepare um "Jjapaguri" para seu filho, e no desdobramento da história a personagem da governanta procura na internet do que se trata e começa a preparar o prato, que consiste na mistura de dois tipos de macarrão instantâneo de marcas coreanas. Curiosamente, a cena apresenta de forma tímida o product placement ${ }^{18}$, apesar de não ter nenhuma confirmação contratual publicitária para inserção do produto no filme, faz se entender a inserção uma vez que são de conhecimento popular as versões do macarrão da marca Nongshim. Fato é que diversos momentos do filme renderam diversas postagens na internet, e a receita de como preparar o prato em um dos momentos de tensão do filme também se tornaram tendência entre os netizens ${ }^{19}$.

A empresa Nongshim responsável pelos produtos produziu duas peças publicitárias para resgatar a lembrança dos seus produtos, se aproveitando da linguagem utilizada no poster do premiado filme, a primeira peça é uma paródia colocando o mascote e produtos da marca como personagens da história, a segunda peça é uma receita de como preparar o prato consumido no filme utilizando os produtos da marca. Ambas as peças foram divulgadas no dia seguinte à cerimônia de entrega do Oscar ao filme. Nota-se que as peças foram divulgadas em língua inglesa, o que nos leva a crer na estratégia da marca de se fortalecer para o público além do seu país.

O consumo do produto apresentado no filme também foi publicado nas redes sociais do embaixador dos EUA na Coréia do Sul, Harry Harris elogiou as premiações do filme e ao final de seu tweet enalteceu o cinema "ROK” (Republic Of Korea), e no mesmo dia a empresa CJ ENM responsável pela distribuição do filme, e parte de um dos maiores conglomerados da Coreia do Sul também divulgou que no dia da premiação incluiu em seu cardápio corporativo a reprodução do prato demonstrado no filme.

\footnotetext{
${ }^{18}$ Técnica publicitária de inserção de produtos dentro de produtos visuais que não possuem inicialmente seu viés publicitário.

19 Termo citado por Philip Kotler como abreviação para "internet citizen", em portugês "cidadão da internet".
} 
Desde o product placement no filme, para a propaganda, passando pela comemoração por conglomerados e embaixadores estadunidenses são apenas um exemplo de um produto inserido no entretenimento que representa uma parcela do avanço da onda coreana a patamares históricos.

No Brasil, o avanço da onda coreana observado através do k-pop, que historicamente tem apresentado uma força comercial diante o histórico de eventos e shows de grupos coreanos como é o caso do grupo BTS e seu público de 89 mil pessoas para dois dias de show. A mobilização de um público grande para um concerto, atuação do público responsável por distribuir as mídias de entretenimento, junto ao alcance a patamares globais do cinema coreano levantou o questionamento de quais influências a cultura exerce a níveis de consumo no Brasil.

A partir do modelo de análise de conteúdo (BARDIN, 1997), a pesquisa se propôs a utilizar o modelo de pesquisa descritiva através de um questionário que utilizam palavras de cunho relacionado ao assunto para fazer surgir associações relativas ao consumo próprio de produtos, a partir da pesquisa foi possível ordenar as palavras de maior recorrência junto a aproximações semânticas de produto e categoria. Segundo Bardin, se prevê no teste que palavras indutoras acarretam em várias palavras induzidas em cadeia, onde podemos orientar a análise para as estruturas de encadeamento de associação, dessa forma o questionário propôs perguntas com relação ao tema, para um grupo de pessoas que possuem um certo nível de conhecimento prévio com produtos da hallyu não sendo de caráter exploratório à todos os públicos. Apesar disto, a divulgação foi realizada pelo Twitter com apoio de páginas com alto número de seguidores, a postagem obteve o alcance de 366r visualizações.

A pesquisa feita em duas etapas buscou qualificar o tipo de conteúdo preferido dos indivíduos, compras de produtos e preferências por categorias; a segunda parte estratificou os indivíduos por região, faixa etária e se são responsáveis financeiros pelas suas próprias despesas, a fim de entender a profundidade de decisão de compra.

Dentre as opções, os clipes e performances musicais foram os preferidos pelos participantes, para além disso os participantes indicaram quais outros conteúdos consomem além do $k$-pop, e classificando os itens por maior expressão de escolha, o 
ranking fica em primeiro lugar os $k$-dramas, em segundo lugar empatado cinema e $k$ food, em terceiro $k$-beauty. Os clipes musicais e $k$-dramas foram os itens que tiveram maior destaque na primeira parte da pesquisa no que se refere a representatividade de respostas, mostrando que na gama de produtos o audiovisual tem uma maior presença na preferência de consumo dos fãs da cultura pop coreana.

Os participantes foram questionados se já fizeram a compra de algum produto por conta da influência de um artista de $k$-pop ou que remetesse ao $k$-pop, e $58 \%$ dos participantes responderam que já tomaram esse tipo de decisão de compra, optando por descrever um breve relato de quais produtos, serviços e marcas haviam comprado. No detalhamento sobre compras relacionadas a hallyu, foram observadas a presença de quatro categorias: música ( $k$-pop), comida ( $k$-food), cosméticos ( $k$-beauty), e uma categoria extra não mapeada, a de vestuário ( $k$-fashion).

Quadro (I)

\begin{tabular}{|l|l|}
\hline Categoria & Palavras-chave \\
\hline K-Pop & Album, photocards, poster, Polaroid \\
\hline K-Food & $\begin{array}{l}\text { Miojo, lámen, comida, chocolate, soju, cerveja, Chapaghetti, Neoguri, } \\
\text { Pepero, Cold Brew, Nutella }\end{array}$ \\
\hline K-Beauty & $\begin{array}{l}\text { Maquiagem, máscara facial, tinta para cabelo, lip tint, perfume, } \\
\text { amaciante, Nature Republic, Tony Moly, BT2I, Bulgari, Downy }\end{array}$ \\
\hline K-Fashion & All Star (Converse), Adidas, camiseta, tênis, tie dye \\
\hline
\end{tabular}

Elaborado pela autora

A categoria " $k$-food" envolvem respostas mais detalhadas, e que relatam a experimentação ao produto a partir da curiosidade advinda dos meios audiovisuais consumidos, um dos participantes relata: "Comprei os miojos coreanos Chapagetti e Neoguri porque vejo os idols/atores comendo em programas/dramas direto [...]" outro participante também afirma: "O próprio miojo, fui influenciada só porque eles vivem comendo". Do grupo que se dispôs a descrever as compras, 31,03\% citam algum alimento ou bebida, em sua maioria produtos industrializados citando marcas coreanas. 
É possível observar como o contato com as marcas coreanas possuem relevância na validação da experimentação, uma vez que nenhum relato cita ir a um restaurante local que sirva comida coreana, ou qualquer outra experiência não mediada por marcas. Isso leva a refletir sobre o papel da industrialização, e de como esses produtos estão chegando primeiro no referencial do público por meio da mídia, que analisando pela ótica de Canclini não se tratam de meras posses de consumo individual isolada, mas uma apropriação simbólica de solidariedade e distinção com outros, onde o valor mercantil não está naturalmente sendo questionado, mas sim um resultado de interações socioculturais, fazendo a ponte entre hábitos culturais e alimentares diferentes da matriz dos indivíduos.

A categoria de roupas e calçados não foi contemplada dentro das opções múltipla escolha como " $k$-fashion", mas curiosamente esse segmento esteve presente em 22,4\% das respostas, e também tiveram citações à marcas como Adidas, Converse, além de marcas criadas pelos próprios artistas.

A forma no qual encontramos os elementos da Hallyu hoje, é possível compreender que o fenômeno não se trata mais de uma novidade, uma vez que se dispensam apresentações do que se trata por meio de grandes mídias que já consideram estes elementos pertencentes ao consumo globalizado do entretenimento. Dentre todos os elementos pertencentes à Hallyu, com exceção de $k$-beauty e $k$-food é possível consumir toda gama de produtos independente da localização do consumidor, inclusive é possível observar que a participação desses elementos coroa toda uma cadeia de consumo levando à experimentação e tangibilização do consumo da Hallyu.

\section{RESULTADOS}

Considerando a primeira hipótese de que o $k$-pop possui potencial de venda indireto para o comércio de produtos coreanos uma vez que são assimilados ao contexto da Hallyu, o palpite inicial era de que os consumidores estariam na faixa etária até I7 anos, curiosamente a pesquisa revela que a maior parte da amostra já são jovens adultos, sendo $53 \%$ de 18 à 24 anos, e $40 \%$ dos 25 aos 30 anos de idade. 
O número expressivo de participantes que, entre outros produtos da hallyu, assinalou os $k$-dramas como a mídia mais consumida nos remete ao início da onda coreana que se deu justamente pela exportação desse produto, indicando que seria um grande equívoco considerar apenas o $k$-pop como o único elemento protagonista na influência cultural.

Por fim, o entendimento de que a hallyu é composto de obras audiovisuais e musicais que atrai o público com narrativas que criam um afeto que excede as barreiras culturais (CHIN; MORIMOTO, 2013) mas tornam seus símbolos culturais e artísticas a porta de entrada não somente da cultura coreana mas também promotor da indústria coreana como um todo, onde mercadologicamente a identidade dos " $k$-produtos" se torna uma espécie de patente cultural, e que garantem a Coréia do Sul direito de exclusividade sobre a produção de uma demanda que eles mesmos criaram. Ainda que sua estrutura possa ser replicada industrialmente por outros países, as condições que determinam o sucesso dependem de uma série de fatores políticos, sociais e principalmente culturais que são intrínsecos à Coreia do Sul.

Por meio da pesquisa foi possível compreender a partir dos relatos que os produtos industrializados, comidas, e costumes promovidos proporcionam uma vivência emocional com seus consumidores, como forma de experimentação do contato com a cultura coreana. Pelos aspectos apresentados, salientamos o potencial de influência da cultura pop coreana no consumo de produtos pelo recorte na publicidade, e cabe a nós observar os desdobramentos diplomáticos e comerciais entre os dois países a partir de uma perspectiva favorável do público brasileiro.

\section{REFERÊNCIAS}

BARDIN, Laurance. Análise de Conteúdo. Tradução de Luis Antero Reto e Augusto Pinheiro. 2a Edição. São Paulo: Martins Fontes, 1979.

BOK-RAE, Kim. Past, Present and Future of Hallyu ( Korean Wave). American International Journal of Contemporary Research Vol.5 n. 5; October 2015. Disponível em:

〈https://pdfs.semanticscholar.org/6c8f/ao5ae6ae253dc618441710bed2e8742c5098.pdf〉 Acesso em: 17 out. 2020 
CANCLINI, Nestor García. Consumidores e Cidadãos: Conflitos Multiculturais da globalização. 3ํ Edição. Rio de Janeiro: UFRJ, 1997.

CHIN, Bertha; MORIMOTO, Lori Hitchcock. Towards a theory of transcultural fandom. Journal of Audience \& Reception Studies, I0:I, 92-108. May 2013. Disponível em:

<https://www.researchgate.net/publication/269763199_Towards_a_Theory_of_Trans cultural_Fandom> Acesso em: 20 out. 2020

CORTEZ, Krystal. Beyond Western Pop Lenses: $O$ circuito das japonesidades e coreanidades pop e seus eventos culturais/musicais no Brasil. Dissertação (Doutorado em Comunicação) In: VII ENCONTRO DE ESTUDOS COREANOS - USP. São Paulo, 2018

GI. BTS abre data extra de show no Brasil após ingressos se esgotarem rapidamente Disponível em: <https://gi.globo.com/pop-arte/musica/noticia/2019/o3/12/bts-abredata-extra-de-show-no-brasil-apos-ingressos-se-esgotarem-rapidamente.ghtml>

Acessado em: 19 jul. 2020

HE-RIN, Jo. Nongshim ups promotion of 'ram-don' from 'Parasite'. Korea Herald. Disponível em : <http://www.koreaherald.com/view.php?ud=20200211000702>Acesso em: 13 jan. 2021

HERMAN, Mario; PENTEK, Tobias. OTTO, Boris. Design principles for industrie 4.0 Scenarios: A Literature Review. Disponível em: 〈https://www.researchgate.net/publication/307864150_Design_Principles_for_Indust rie_40_Scenarios_A_Literature_Review> Acesso em: I6 fev. 2021

JENKINS, Henry. Cultura da Convergência. Tradução de Suzana L. de Alexandria. 2" Edição. São Paulo: Aleph, 2009.

JOO, Jeongsuk. Transnationalization of Korean popular culture and the rise of "pop nationalism" in Korea . The Journal of Popular Culture, 44: 3, 489-504. 201 .

KOTLER, Philip; KARTAJAYA, Herman; SETIAWAN, Iwan. Marketing 3.o: as forças que estão definindo o novo marketing centrado no ser humano. Tradução de Ana Beatriz Rodrigues. Edição digital ePub. Rio de Janeiro : Elsevier, 2012.

KOTLER, Philip; KARTAJAYA, Herman; SETIAWAN, Iwan. Marketing 4.o: Do tradicional ao digital. Tradução de Ivo Korytowski. Edição digital ePub. Rio de Janeiro: Sextante, 2017.

KOTLER, Philip; KELlER, Kevin Lane. Administração de Marketing. I2 ed. São Paulo: Pearson Prentice Hall, 2006. 
KYOUNG-SON. Song Nongshim ups promotion of 'ram-don' from 'Parasite'. Korea JongAng Daily in association with The New York Times. In fev. 2020. Disponível em: $<$ https://koreajoongangdaily.joins.com/2020/02/II/industry/Its-as-if-the-chapagurinoodles-won-the-Oscar/3073706.html> Acesso em I3 jan. 2021

MINNS, John. Of miracles and models: the rise and decline of the developmental state in South Korea. Third World Quarterly, Vol 22, n6, pp 1025-1043, 2001. Disponível em: 〈https://library.fes.de/libalt/journals/swetsfulltext/r2303107.pdf 〉 Acesso em I9 out. 2020

MAZUR, Daniela. Um Mergulho na Onda Coreana, Nostalgia e Cultura pop na série de K-dramas "Reply". Dissertação (Mestrado em Comunicação) In: VII ENCONTRO DE ESTUDOS COREANOS - USP. São Paulo, 2018

NYE, Joseph. Soft Power: the means to success in world politics. New York: Public Affairs, 2004.

SETH, Michael J. A Concise History of Modern Korea: From the Late Nineteenth Century to the Preset. Editora: Rowman \& Littlefield Publishers, 2009. Versão ebook.

VINCO, Alessandra. Além do 'Gangnam Style': Relações globais, autenticidade e fãs de K-pop no Brasil. In: VII ENCONTRO DE ESTUDOS COREANOS - USP. São Paulo, 2018

VINCO, Alessandra; MANZUR, Daniela; URBANO, Krystal. Fãs, Mediação e Cultura Midiática: Dramas Asiáticos no Brasil. In: 2014, I Jornada Internacional GEMInIS: Entretenimento Transmídia. Disponível em <https://www.academia.edu/9152520/F\%C3\%A3s_Media\%C3\%A 7\%C3\%A30_e_Cult ura_Midi\%C3\%Artica_Dramas_Asi\%C3\%Articos_no_Brasil> Acessado em: io jun. 2020 